\title{
Antizyklische Kapitalpuffer und Kreditvergabe
}

\author{
Linda Kirschner
}

\section{Relevanz}

Banken pflegen mit ,ihren“ Unternehmen enge Geschäftsbeziehungen und können ihnen mit stabiler Kreditfinanzierung über schwierige Perioden hinweghelfen. Dazu brauchen sie eine solide Kapitalausstattung und müssen in Phasen des Booms, wenn sie gut verdienen, Reserven aufbauen, auf die sie in einer Rezession zurückgreifen können. So gelingt es ihnen, für ihre Firmenkunden eine stetige Kreditfinanzierung sicherzustellen und für die Gesamtwirtschaft den Abschwung abzumildern, damit weniger Jobs vernichtet werden. Auf diesem Weg können ein gut kapitalisierter Bankensektor und eine Regulierung mit antizyklischen Kapitalpuffern wesentlich zur wirtschaftlichen Sicherheit und zu stabilem Wachstum beitragen.

Christian Keuschnigg

\section{Quelle}

Der nachfolgende Text ist eine Zusammenfassung von: Jiménez, Gabriel, Steven Ongena, José-Luis Peydró und Jesús Saurina, Macroprudential Policy, Countercyclical Bank Capital Buffers and Credit Supply, Journal of Political Economy 125, no. 6 (December 2017): 2126-2177.

L. Kirschner $(\bowtie)$

Universität St. Gallen, St. Gallen, Schweiz

E-Mail: linda.kirschner@unisg.ch

(C) Der/die Autor(en) 2018

C. Keuschnigg (Hrsg.), Inklusives Wachstum und wirtschaftliche Sicherheit, https://doi.org/10.1007/978-3-658-21344-2_26 
Bankenkrisen sind keine Seltenheit. Sie treten meist nach längeren Perioden des Booms mit starkem Kreditwachstum ein und verschärfen die Rezession. Angesichts der negativen Auswirkungen auf die Realwirtschaft besteht Konsens zwischen Wissenschaft und Politik, dass die Regulierung des Finanzsystems auch zur makroökonomischen Stabilisierung (makro-prudenzielle Regulierung) beitragen soll, um negative Folgen einer Kreditklemme auf die Realwirtschaft zu vermeiden. Nach Basel III sind antizyklische Kapitalpuffer vorgesehen, wonach die Banken in Phasen des Booms, wo sie gut verdienen, zusätzliche Kapitalreserven bilden müssen, die sie in der Rezession wieder abbauen können. Dieser Mechanismus trägt dazu bei, kreditgetriebene Boomphasen etwas abzukühlen, und sorgt in der Rezession für Entspannung. Antizyklische Kapitalpuffer sollen dazu beitragen, die Kreditvergabe der Banken über die Zeit zu glätten und damit die Konjunkturschwankungen zu stauchen.

Die Forscher versuchen, den Effekt eines solchen antizyklischen Kapitalpuffers auf das Kreditangebot und auf die realwirtschaftliche Aktivität zu quantifizieren. Konkret studieren sie, wie sich die Einführung dynamischer Kapitalreserven als Instrument der Risikovorsorgebildung in Spanien ausgewirkt hat. Dieses regulatorische Instrument ist im Wesentlichen mit einem antizyklischen Kapitalpuffer vergleichbar. Die spanische Zentralbank schreibt den regulierten Banken die Bildung von Rückstellungen nach einer statistischen Formel vor, welche auf zwei Komponenten abstellt, nämlich dem Volumen der Kreditvergabe und den zu erwarteten Wertminderungen relativ zum Durchschnitt des vergangenen Kreditzyklus. Für die Banken ergeben sich daher unterschiedliche Rückstellungen je nach Umfang und Struktur ihres Kreditportfolios. Wenn sie im Boom ihr Kreditvolumen ausweitet, muss sie mehr Rückstellungen bilden, wenn in einer Rezession das Kreditvolumen sinkt, dann weniger. Die andere Komponente hängt von den notwendigen Abschreibungen und damit von der Qualität ihres Kreditportfolios ab. Bei gutem Wirtschaftsgang gibt es wenig notleidende Kredite, so dass die Bank nur wenige Wertberichtigungen vornehmen muss, weniger als im Durchschnitt der letzten Jahre. Dann muss sie überdurchschnittlich hohe Rückstellungen bilden, um für die nächste Rezession vorzusorgen, und kann dies angesichts des guten Geschäftsgangs leicht tun. In einer Rezession nimmt die Anzahl notleidender Kredite zu, die Wertberichtigungen fallen relativ zur vergangenen Periode überdurchschnittlich hoch aus, und die Bank macht zudem wenig Gewinne. In diesem Fall schreibt die Formel nur geringe Rückstellungen vor und die Bank kann von ihren vorher aufgebauten Reserven zehren. Diese dynamische Risikovorsorgebildung ist somit ein vorwärts gerichtetes und stabilisierendes Instrument der Regulierung, da die Banken während guter Phasen höhere Rückstellungen 
zwecks Risikovorsorge bilden oder in Vorsorgefonds ${ }^{1}$ einzahlen müssen und in Krisenzeiten diese Rückstellungen wieder aufzulösen können.

In Spanien müssen Banken dynamische Kapitalreserven bilden. Im Boom werden sie mit hohen Rückstellungen aufgebaut, in der Rezession können Banken darauf zurückgreifen. Ziel ist es, die Kreditvergabe über den Konjunkturzyklus zu stabilisieren.

Die Forscher untersuchen den Einfluss der dynamischen Risikovorsorge auf die Kreditvergabe sowie auf den Kreditzugang der Unternehmen sowie die damit verbundenen Auswirkungen auf die Beschäftigung und Überlebenswahrscheinlichkeit von Firmen. Folgende Mechanismen stehen im Vordergrund. Das regulatorische Instrument wurde in einer Hochphase des Kreditzyklus (2000:Q1-2001:Q2) eingeführt. Die Hypothese ist, dass die von der Regulierung betroffenen Banken ihr Kreditangebot wegen der höheren Kapitalkosten verringern. Die Unternehmen versuchen, Kredite von anderen Banken zu erhalten. Sollte dies fehlschlagen, müssten sie als Folge der Kreditklemme ihre Beschäftigung senken und ihre Überlebensrate würde sinken. Die Regulierung würde damit im Boom etwas dämpfend wirken. Während der Krisenphase (2008:Q1-2010:Q1) konnten die Banken Rückstellungen auflösen, um Finanzierungsengpässe zu überbrücken. Zunächst durften nur $67 \%$ der Rückstellungen aufgelöst werden, im vierten Quartal des Jahres 2008 wurde dies auf $90 \%$ erhöht und Ende 2009 sogar auf $100 \%$. Wiederum untersuchen die Autoren das Kreditangebot sowie die realen Effekte auf den Unternehmenssektor. Um möglichst robuste Ergebnisse zu erzielen, kontrollieren die Forscher für zeitlich variierende Banken- und Firmencharakteristika, die neben der Regulierung ebenfalls Einfluss auf die Ergebnisse haben könnten. Sie nutzen den Datensatz der spanischen Zentralbank über die Vorsorgebildung der einzelnen Banken sowie Daten aus einem umfassenden Kreditregister, das alle nationalen Bankenkredite während eines Kreditzyklus aufzeichnet und sowohl Firmen- als auch Bankbilanzdaten zuordnet. Sie untersuchen Gewerbe- und Industriedarlehen von Geschäftsbanken, Sparkassen und Kreditkooperativen an Gesellschaften mit beschränkter Haftung. Der Datensatz umfasst $80 \%$ der gesamten Kredite von über 175 Banken an 100.000 Unternehmen.

In der Hochphase während der Einführung der Regulierung stellt man eine erhebliche Streuung der dynamischen Rückstellungen für die Risikovorsorge

${ }^{1}$ Dynamische Risikovorsorgefonds zählen zum Tier-2 regulatorischen Kapital. 
zwischen den Banken fest. Der Durchschnitt liegt bei 0,26\% der gesamten Vermögenswerte mit einer Standardabweichung von 0,1\%. Leider geben die Daten keinen Aufschluss darüber, wie der Vorsorgebeitrag bezüglich der Bankcharakteristika schwankt. Allerdings beobachten die Autoren, dass Banken mit einer niedrigeren Liquiditätsquote höhere Risikobeiträge leisteten. In der ersten Zeitspanne der Hochphase ist der geschätzte Koeffizient für den dynamischen Vorsorgebeitrag statistisch signifikant. Eine Erhöhung um eine Standardabweichung $(0,1 \%)$ reduziert die zugesagten Kredite um 4 Prozentpunkte. Dieser Effekt ist ökonomisch durchaus relevant, da die zugesagten Kreditlinien zwischen 2000:Q1 und 2001:Q2 im Durchschnitt um $2 \%$ auf schrumpften.

In der Hochphase führt eine Erhöhung von Rückstellungen für die dynamische Risikovorsorge um 0,1 \% zu einem Rückgang des Kreditangebots um 4 Prozentpunkte. Negative Auswirkungen auf die Realwirtschaft bleiben weitgehend aus.

Auf Unternehmensebene würde man intuitiv erwarten, dass diese Reduzierung des Kreditangebots die Finanzierungsmöglichkeiten einschränkt. Dies könnte abgemildert werden, wenn die Unternehmen auf Kredite von anderen Banken ausweichen könnten, die weniger von der Regulierung betroffenen sind. Die Analyse zeigt, dass genau dies in Spanien der Fall war. Es ist in dieser Hochphase kein statistisch signifikanter Effekt auf die Vermögenswerte, den Beschäftigungsgrad und die Überlebensrate der Unternehmen zu beobachten. Man kann also gut davon ausgehen, dass die dynamische Risikovorsorge während guter Zeiten die Banken dazu veranlasst, ihre Kreditvergabe zu senken, allerdings nur bei den betroffenen Banken. Nennenswerte Einschränkungen bei den Unternehmen sind nicht zu erwarten.

Andere Ergebnisse erhalten die Autoren bei der Untersuchung des Zeitraums nach Eintreten der unerwarteten Kreditkrise nach 2008, als die Rückstellungen aufgelöst und die Reserven langsam abgebaut wurden. Die Autoren nutzen die Daten über den tatsächlichen Risikovorsorgepuffer, den die Banken kurz vor dem Ausbruch der Krise aufgebaut haben. Diese Variable „Dynamic Provision Funds" schwankt stark zwischen den einzelnen Banken und weist über den Zeitraum einen Durchschnittswert von $1,17 \%$ der gesamten Vermögenswerte mit einer Standardabweichung von $0,23 \%$ auf. Der daraus geschätzte Koeffizient ist statistisch und ökonomisch signifikant. Wenn die Beiträge der Bank vor der Krise um eine Standardabweichung höher lagen, dann erlaubt dies ein um 8 Prozentpunkte höheres Kreditwachstum während der Krisenperiode. Eine Bank mit einem durchschnittlichen Risikovorsorgepuffer von 1,17 \% ihrer Vermögenswerte 
verzeichnete ein um fast 41 Prozentpunkte höheres Kreditwachstum als eine Bank ohne einen solchen Kapitalpuffer.

\begin{abstract}
Eine Erhöhung des Vorsorgefonds um 1 Prozentpunkt vor der Krise erhöht in der folgenden Rezession die zugesagten Kredite um 9 Prozentpunkte, das Beschäftigungswachstum um 6 Prozentpunkte und die Überlebensrate der Unternehmen um 1 Prozentpunkt.
\end{abstract}

Die Kapitalregulierung hat Folgen für die von den Banken finanzierten Unternehmen. Unternehmen, deren Banken vor der Krise 1 Prozentpunkt mehr Kapital im Vorsorgefonds hielten, erhalten 9 Prozentpunkte mehr an zugesagten Kreditfazilitäten. In der Krise kam es zu einem durchschnittlichen Rückgang von zugesagten Krediten um $34 \%$. Banken mit einem hohen Kapitalpuffer konnten damit die Krise etwas entschärfen und die Lage in den von ihnen finanzierten Unternehmen wenigsten zum Teil stabilisieren. Das Beschäftigungswachstum in den Unternehmen, deren Banken mit hohen Kapitalreserven ausgestattet waren, entwickelte sich wesentlich positiver als in anderen Unternehmen. Der geschätzte Koeffizient von 0,056 bedeutet ein knapp 6 Prozentpunkte stärkeres Wachstum für jeden zusätzlichen Prozentpunkt an Fondsvermögen vor der Krise. Der durchschnittliche Einbruch der Beschäftigung lag in dieser Krisenperiode bei -14\%. Das höhere Wachstum konnte diesen starken Rückgang wenigstens teilweise ausgleichen. Die Auswirkung auf die Überlebensrate der Unternehmen ist ähnlich. Ein um 1 Prozentpunkt höherer Puffer führte zu 1 Prozentpunkt höherer Überlebenswahrscheinlich-keit. Im Durchschnitt wurden während der Krisenperiode $6 \%$ der Unternehmen zahlungsunfähig.

Open Access Dieses Kapitel wird unter der Creative Commons Namensnennung 4.0 International Lizenz (http://creativecommons.org/licenses/by/4.0/deed.de) veröffentlicht, welche die Nutzung, Vervielfältigung, Bearbeitung, Verbreitung und Wiedergabe in jeglichem Medium und Format erlaubt, sofern Sie den/die ursprünglichen Autor(en) und die Quelle ordnungsgemäß nennen, einen Link zur Creative Commons Lizenz beifügen und angeben, ob Änderungen vorgenommen wurden.

Die in diesem Kapitel enthaltenen Bilder und sonstiges Drittmaterial unterliegen ebenfalls der genannten Creative Commons Lizenz, sofern sich aus der Abbildungslegende nichts anderes ergibt. Sofern das betreffende Material nicht unter der genannten Creative Commons Lizenz steht und die betreffende Handlung nicht nach gesetzlichen Vorschriften erlaubt ist, ist für die oben aufgeführten Weiterverwendungen des Materials die Einwilligung des jeweiligen Rechteinhabers einzuholen.

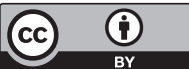

